\title{
La Gestión de la Calidad y las Necesidades Académicas en Deportistas Calificados de la UNMSM 2017 - 2019
}

\author{
Quality Management and Academic Needs in UNMSM \\ Qualified Athletes 2017 - 2019
}

\begin{abstract}
RESUMEN
El artículo desarrollado permitió reconocer y analizar las dificultades encontradas en la administración deportiva, específicamente en la gestión del apoyo académico para el atleta - estudiante. Aquí se pudo comprobar la alta tasa de deserción estudiantil durante los años 2017 al 2019, así mismo se realizó un estudio descriptivo, mediante recopilación de información histórica, análisis de reportes y documentación oficial del departamento de planificación de la Universidad Nacional Mayor de San Marcos (UNMSM). En esta investigación se demostró que existe desconocimiento en la aplicación de estrategias para los apoyos académicos, además que, a través del manejo adecuado del modelo Malcolm Baldrige de la calidad, destinado a cubrir los requerimientos estudiantiles y utilizando métrica e indicadores adecuados, se puede lograr la mejora continua buscando la satisfacción de las necesidades académicas en atletas - estudiantes calificados.
\end{abstract}

Palabras claves: Modelo Malcolm Baldrige; administración deportiva; gestión de la calidad; deportistas calificados.

\begin{abstract}
The developed article focuses on the ability to recognize and analyze the problems found in Sports Administration Management, specifically in the management for the academic support of the qualified student-athlete. It was possible to verify the high rate with student desertion in the athletes of UNMSM during the years 2017 - 2019. A descriptive study is also carried out through the collection of historical information, analysis of indicators in reports and official documentation of the planning department of UNMSM. In this research, it is demonstrated that there is a total lack of knowledge about the management of academic support for the qualified athlete. Through the adequate management of the Malcolm Baldrige model of quality, one can cover the student's requirements using appropriate indicators, and can achieve continuous improvement by meeting the academic needs of qualified student-athletes.
\end{abstract}

Keywords: Malcolm Baldrige model; sports administration; quality management; qualified athletes.

Presentado: 25/10/2019 - Aceptado: 02/06/2020 - Publicado: 20/10/2020

(C) Los autores. Este artículo es publicado por Gestión en el Tercer Milenio de la Facultad de Ciencias Administrativas de la Universidad Nacional Mayor de San Marcos. Este es un artículo de acceso abierto, distribuido bajo los términos de la licencia Creative Commons Atribucion - No Comercia_Compartir Igual 4.0 Internacional. (http://creativecommons.org/licenses/by-nc-sa/4.0/) que permite el uso no comercial, distribución y reproducción en cualquier medio, siempre que la obra original sea debidamente citada. 


\section{INTRODUCCIÓN}

El deporte universitario en el Perú tiene alrededor de ochenta años, el encargado del aspecto normativo es la FEDUP (Federación Deportiva Universitaria del Perú), la cual fue fundada el 07 de agosto de 1924 en la Universidad Nacional Mayor de San Marcos (UNMSM). A pesar de que cumple un papel protagónico e integrante del SISDEN (Sistema Deportivo Nacional) es un filtro para la detección y mantenimiento de potenciales deportistas en las distintas disciplinas olímpicas para las selecciones nacionales, en este caso los campeonatos nacionales universitarios que cambian de sede cada dos años, normalmente, no tienen acogida mediática y no se le da la importancia debida que corresponde. Cabe recordar que la labor de la UNMSM es desarrollar la actividad deportiva como medio de formación integral y complementaria a la vida académica del estudiante, sin embargo, por lo observado, el modelo de administración estatal burocrática que se aplica no suele ser dinámico en la capacidad de reacción ante los constantes cambios del entorno, requiere siempre de personal adecuado o especializado que cubra los puestos claves en las unidades estratégicas, en ese sentido se puede afirmar que desde hace mucho tiempo permanece inamovible hacia la mejora del ecosistema deportivo universitario.

La perspectiva orientada al problema se convierte en una oportunidad para desarrollar la llamada "dualidad de carrera", que en países desarrollados ya es una realidad, la formación de un deportista de alta competencia y a la vez egresado de una carrera profesional en la universidad, esto quiere decir, especializar la práctica del deporte, crear auto exigencia académica y lograr competitividad. Sin embargo, en la realidad local, la exigencia en la parte académica, la participación deportiva que es simultánea al ritmo de clases y actividades estudiantil, ejercen en el propio atleta - estudiante un nivel de presión hasta el punto de hacerlo decidir entre el formarse profesionalmente o continuar con la práctica deportiva, comprometiendo su futuro tanto académico como deportivo.

El proceso de crear conciencia a nivel de la comunidad universitaria sobre la importancia del deporte es aún la tarea pendiente, persisten los prejuicios que inclusive en la misma universidad encapsulan el concepto del deportista-estudiante de considerarlo de muy bajo nivel académico, o personas que ingresan a la universidad solo para pasar el tiempo en un gimnasio y sin ningún beneficio visible para la universidad.

Desde aquí inicia un análisis multinivel y multidisciplinario que si se entiende en otras latitudes sobre todo en países más desarrollados en materia de Gestión deportiva como en Estados Unidos, Canadá, Brasil, México y Colombia. Sobre la labor en el apoyo al estudiante-atleta, el cual merece un trato no solamente más humano sino más profesional alrededor de la actuación que realizan anualmente y que se traduce en ciclos deportivos que incluyen sesiones desgastantes de entrenamientos intensivos, alimentación estricta y balanceada, suplementación vitamínica con seguimiento de un especialista, entrenamiento silencioso traducido en descanso pasivo. Sobre todo apoyo tanto en la parte logística deportiva como académica para poder lograr el objetivo final al culminar los estudios de manera exitosa, tomando en cuenta los resultados del proceso como un indicador de logro. (Ver Figura 3)

En relación con los problemas entre los programas académicos y las necesidades de los deportistas de alto nivel Echeita y Duk (2008) aseguran:

La inclusión educativa es una aspiración a nivel Iberoamericano si se quiere llegar a tener una educación que realmente contribuya al desarrollo de una sociedad justa, democrática y solidaria. Este interés de hablar acerca de la inclusión a nivel educativo se da al detectar la desigualdad en el sistema educación, las deficiencias y limitaciones del propio, pues no consideran la totalidad de las necesidades de los estudiantes. (p. 1)

En un Estado de derecho y democracia es presumible que todo ciudadano merece recibir la educación igualitaria que se propugna tanto en la Constitución Política del Estado, los lineamientos del Ministerio de Educación y los estatutos de la UNMSM. Estos coinciden en señalar que todos los alumnos deben contar con iguales oportunidades de acceder tanto al ingreso, permanencia y culminación de los 
estudios indistintamente de la condición del estudiante. En este caso al no contar con un sistema eficiente de apoyo al deportista que representa, compite y logra éxitos deportivos en nombre de la universidad a la cual ingresa, no se justifica dejarlo de lado en su derecho por egresar de la carrera elegida. Además, están también justificadas las ayudas referentes a su incursión en el mercado laboral, por ende, el no permitir o facilitar la culminación de estudios superiores a un deportista calificado estudiante universitario significaría atentar contra su derecho fundamental de formarse académicamente inclusive discriminando socialmente de manera indirecta.

En este contexto, la situación problemática del deporte en la UNMSM se agrava debido a que se observó el detrimento en el número de ingresantes en la modalidad de deportistas calificados de alto rendimiento, otro punto que debe ser analizado en subsiguientes estudios. Por otra parte, se recoge la manifestación de los deportistas calificados quienes en reiteradas oportunidades y en base al diálogo permanente establecido durante los últimos meses del año 2018, afirmaron que mantienen un estricto régimen de preparación que incluyen viajes y calendarios competitivos para representar a la selección nacional de la disciplina en la que se desarrollan. Incluso, si representan a la universidad en la que estudian y si pertenece a los registros de algún club federado, deben representarlos en los tres casos de manera obligatoria. Para ellos, esto representa la principal dificultad para seguir los estudios universitarios de manera regular. Así mismo, se procedió a extraer información tanto de la Oficina de Deportes de la UNMSM como la Unidad de Bienestar Universitario de la UNMSM, sin embargo, las oficinas afirmaron que se desconoce data estadística precisa y de estudios multidisciplinarios para consensuar sobre la problemática referida al nivel de deserción estudiantil.

En relación también a esta problemática es importante el aporte de Terragosa, Sanchez, y Cruz (2004) quienes afirman:

El desarrollo de programas para facilitar la compaginación de la carrera deportiva con el desarrollo académico vocacional constituye una herramienta muy importante para facilitar la transición de la vida deportiva a la vida profesional, dichos programas de asesoramiento requieren de una aplicación individualizada para cada deportista en función de sus necesidades y las del deporte en que compiten. (p. 226)

\section{La administración del deporte.}

Según lo afirmado por Bravo (2013), quien considera que el deporte como parte de estudio, necesita de varios niveles de organización del entorno, el deporte organizado necesita de estados, procesos, causas y efectos que interactúan también en los sistemas de las sociedades. Por lo tanto, para lograr una comprensión objetiva de lo que ocurre con el fenómeno del deporte en sí, la investigación en el deporte debe decantarse hacia varios niveles de entendimiento, orientada y explicada multidisciplinariamente. Tal como afirma Dorado (2008) que aportó interesantes propuestas en el congreso de AGESPORT (Asociación Andaluza de Gestores del Deporte) en Andalucía, explicando de manera precisa el valor de la calidad para lograr mejores instituciones deportivas en el tiempo, gestionar a través de la calidad le supone a las organizaciones evolucionar en función de las exigencias y requerimientos de los usuarios o clientes.

En ese contexto, es importante el aporte de Terragosa, Sanchez y Cruz (2004) quienes afirman:

El desarrollo de programas para facilitar la compaginación de la carrera deportiva con el desarrollo académico vocacional constituye una herramienta muy importante para facilitar la transición de la vida deportiva a la vida profesional, dichos programas de asesoramiento requieren de una aplicación individualizada para cada deportista en función de sus necesidades y las del deporte en que compiten. (p. 226)

\section{El concepto de la calidad.}

Según Egaña (2014) en torno a la calidad existen múltiples y diversos conceptos de lo que es la calidad, como por ejemplo el "cero defectos" como lo define Crosby (1979) o "dar satisfacción 
al cliente" de Deming (1989). Como es el caso de Gallardo y Jiménez (2004), quienes refieren otra definición interesante de Tamanes (2000), quien da un significado a la calidad como "un conjunto de rasgos característicos de un producto o servicio, que lo hacen adecuado para satisfacer las necesidades del consumidor o usuario".

\section{Descripción del modelo de excelencia en la gestión Malcolm Baldrige.}

Este modelo de gestión de la calidad es una herramienta creada con la intención de evaluar, planificar y preparar a las organizaciones en su camino hacia la excelencia. Consta de siete capítulos, en seis de ellos se enfatiza sobre la gestión, el último de ellos tiene que ver con los resultados (United States Department of Commerce, 2005). Se debe aplicar a un cuadernillo con un cuestionario de preguntas que deben ser respondidas por los miembros de la organización que aspira a conseguir la certificación de la calidad y a gestionarse con la calidad como referente (Ver Figura 1).

- Presenta una marcada orientación a resultados.

- Prioriza la importancia de desarrollar alineamiento empresarial.

- Propugna un enfoque orientado hacia los clientes.

- Propugna un enfoque orientado hacia los clientes.

\section{Mecanismo de Evaluación.}

El Modelo Baldrige mantiene un sistema único de evaluación sobre los indicadores. Concretamente afirma que los indicadores deben generar:

- Datos relevantes que demuestren una evolución favorable a largo plazo. De esta forma, se asume que las organizaciones estarán preocupadas en realizar mediciones de manera constante.

- Los indicadores deben ser un "síntoma" de mejora para el sistema. (United States Department of Commerce, 2005).

Las organizaciones deben acumular información que sirva de comparación para cada indicador determinado, dentro de estas comparaciones debe incluirse las metas de la misma organización. Las comparaciones deben comprender las metas propias, el proceso, desempeño y resultados, de esta manera, se asegura de tener un marco de referencia para la mejora continua de la organización. (United States Department of Commerce, 2005)

En el caso puntual del premio a la calidad en el Perú se deben descargar varios documentos desde el portal institucional del Centro de Desarrollo Industrial del Perú (CDI), se accede a las fichas de inscripción y hojas excel conteniendo los cuestionarios y plantillas para la autoevaluación del modelo Malcolm Baldrige con la intención de competir en el premio a la calidad que cada año se pone en juego por esta mencionada institución.

\begin{tabular}{|c|c|}
\hline Liderazgo & 120 \\
\hline Planificación estratégica & 85 \\
\hline Enfoque al diente y mercado & 85 \\
\hline Información y análisis & 90 \\
\hline Enfoque RRHH & 85 \\
\hline Gestión de procesos & 85 \\
\hline Resultados del negocio & 450 \\
\hline
\end{tabular}

Figura 1. Criterios estratégicos del Modelo Malcolm Baldrige y los puntajes para el obtener el premio a la calidad.

Elaboración propia. 
Dorado y Gallardo (2005) afirman en su obra que la cultura define el "sistema de valores" de la organización, los mismos que condicionan las principales decisiones que se adoptan en todos los procesos de gestión en las organizaciones. Los cambios que se produzcan alrededor de la cultura de la organización deben estar sostenidos de arriba hacia abajo, esto implica también una nueva forma de hacer las cosas y gestionar los procesos no solamente adoptar una nueva cultura en los cambios de la organización.

\section{Políticas Deportivas.}

El Deporte en la sociedad actual tiene un significado diferente. Existe una legislación preocupada por el desarrollo de las organizaciones deportivas además de las ya existentes, la última de ellas recientemente aprobada y promulgada el año 2017, Ley $\mathrm{N}$ ㅇ 30746, que regula los programas deportivos de alta competencia en las universidades, aún falta su reglamentación y por parte de las universidades, crear una gestión adecuada para que se ajusten a las directivas de esta. Tiene por finalidad regular el programa deportivo de alta competencia para las universidades peruanas tanto nacionales como privadas, conforme a lo establecido en el artículo 131 de la ley N. o 30220, ley universitaria. Las universidades deben desarrollar un adecuado modelo de gestión de tutorías académicas con el fin de lograr que los deportistas terminen adecuadamente los estudios universitarios que eligieron seguir, indistintamente si es una institución educativa superior nacional o privada.

Antúnez (2000) sostiene que "el estado debe estar a la altura de los tiempos y las exigencias del deporte actual con todos sus cambios, estar a tono con las políticas generales e ir adaptándose a los cambios que se producen", esto refleja la amplitud de análisis del autor en referencia al papel dinámico del estado, que debe tomar en cuenta al establecer políticas a medida de las necesidades del sistema deportivo nacional, siendo dúctil y pragmático, con capacidad de reacción ante los cambios globalizados de los modelos de gestión.

En relación con el aparato estatal, que debe estar compenetrado con el objetivo de trabajar en las políticas públicas inclusivas hacia la sociedad, generar apoyos en los proyectos de desarrollo deportivo. (Meynaud 1972)

- Formación de escuelas deportivas con el fin de capacitar adecuadamente a los encargados de la educación física y el deporte en todo el país.

- Incrementar el presupuesto para investigación en temas relacionados directamente con el desarrollo del deporte nacional (trabajo psicológico, medicina deportiva, higiene escolar, entre otras).

- Construcción de mayor y mejor infraestructura deportiva en el territorio nacional atendiendo a las necesidades reales de la población.

- Concesión para subvencionar la formación de los atletas que representan al país en competiciones nacionales, inclusive en su formación antes del certamen.

- Contribución para los eventos deportivos que se organicen en el país.

- Difusión de las actividades deportivas de paraatletas nacionales.

- Autogestión y autofinanciamiento de las organizaciones deportivas.

- Mejoras en la Gestión de administración deportiva en las instituciones que desarrollan el deporte como complemento a su actividad principal.

- Crear mecanismos que faciliten el ingreso de atletas o deportistas calificados que decidan iniciar estudios superiores universitarios o técnicos.

- Reconocer a los estudiantes deportistas como un individuo de importantísimo valor para la sociedad que toda universidad debe apoyar inclusive más allá del egresar de una carrera universitaria, sino que también debe implantar mecanismos para gestionar su inclusión laboral.

- Generando este tipo de políticas inclusivas lo que se debe buscar principalmente es luchar contra cualquier tipo de encasillamiento social por la naturaleza de ser deportista calificado y no crear estrategias eficientes 
para lograr que egrese satisfactoriamente de sus estudios, se incurriría en una falta relacionada con la discriminación.

La pregunta de investigación es:

¿Cuál es la problemática existente en el sistema de administración deportiva en la UNMSM en relación con el apoyo al deportista universitario y que estrategias eficientes puede aplicar para lograr el acceso, retención, permanencia y finalización de los estudios universitarios en los deportistas de alto rendimiento de la UNMSM entre 2017- 2019?

\section{Hipótesis:}

El actual modelo del sistema de administración deportiva en la Universidad Nacional Mayor de San Marcos desconoce estrategias eficientes de acceso, retención, permanencia y finalización de los estudios universitarios en los deportistas de alto rendimiento de la UNMSM 2017- 2019.

\section{Objetivo:}

Describir la problemática existente en el sistema de administración deportiva en la Universidad Nacional Mayor de San Marcos, y proponer estrategias eficientes para lograr el acceso, retención, permanencia y finalización de los estudios universitarios en los deportistas de alto rendimiento de la UNMSM entre 2017- 2019.

\section{MÉTODOS}

En cuanto al nivel, de acuerdo a la naturaleza del problema planteado y objetivos propuestos, la investigación es de tipo descriptivo porque busca precisar las características de la gestión actual en el sistema deportivo de la UNMSM. Respecto al método empleado, es decir de acuerdo a la estrategia que se utilizó para comprobar las hipótesis formuladas, es una investigación aplicada, de tipo observacional, prospectiva, transversal y explicativa porque tratamos de buscar la explicación del comportamiento de las variables con el fin último de descubrir las causas de la deficiencia administrativa del sistema deportivo en la UNMSM. El diseño metodológico por la naturaleza del estudio es el descriptivo; mediante entrevistas, análisis documental, búsqueda bibliográfica y revisión de documentos estadísticos de memoriales de gestión de los últimos 5 años.

Para el desarrollo de la investigación se inició con la construcción del marco teórico (antecedentes nacionales e internacionales y bases teóricas) necesario para el adecuado conocimiento e interpretación de la problemática actual en el sistema de administración deportiva en la UNMSM en relación con el apoyo académico al deportista calificado y de alto rendimiento (Ver tabla 1).

\section{Tabla 1.}

Deportistas ingresantes / deportistas egresados (20112017)

\begin{tabular}{ccc} 
& Deportistas Calificados UNMSM \\
\hline Año & Total ingresantes & Total Egresados \\
2011 & 121 & 11 \\
2012 & 129 & 9 \\
2013 & 116 & 3 \\
2014 & 163 & 5 \\
2015 & 156 & 13 \\
2016 & 137 & 7 \\
2017 & 117 & 10 \\
Total & 939 & 58 \\
\hline
\end{tabular}

Nota. Elaboración propia en base a información recabada de la Unidad de Planificación de la UNMSM.

\section{RESULTADOS}

Según los datos obtenidos y la contrastación de la información con cada una de las facultades de la UNMSM, se puede precisar que desde el año 2011 hasta el año 2017, del total de deportistas ingresantes en las distintas modalidades existentes en total suman 939, de los cuales hasta el momento solo han egresado 58 de estos, representando el $6 \%$ del total de ingresantes.

Con las cifras que presentamos a continuación, podemos comprobar que el alejamiento de los estudios es sumamente crítico en los atletas-estudiantes de la UNMSM.

Como objetivo de este programa se encuentra el apoyar a los deportistas calificados que cumplan con las especificaciones y requisitos estipulados en la ley universitaria y reglamento de la Oficina de Deportes de forma que puedan armonizar los estudios universitarios con la práctica deportiva; en la cual se estima sesiones de entrenamiento, concentraciones, 
viajes y competiciones entre otras actividades necesarias para la obtención de resultados efectivos esperados en los indicadores de logros deportivos (Ver figura 2).

De esta manera se busca lograr los siguientes objetivos secundarios:

- Comunicar, informar y difundir la información sobre el alcance, características y beneficios del Programa de Apoyo en tutorías entre el alumnado implicado.

- Proponer, Seleccionar y determinar al Docente - tutor que les acompañe durante su carrera deportivo-académica.

- Aplicar los reajustes académicos de ser necesarios para hacer viables y realizables en las mejores condiciones posibles, las exigencias académicas y deportivas de los estudiantes-atletas.

- Crear la mejor opción y el más eficiente diseño de la secuencia de asignaturas, con especial atención a las de carácter no obligatorio.

- Mejorar la adaptación del estudiante-atleta al ámbito académico, teniendo en cuenta que las actividades deportivas pueden crear algunas dificultades en el desarrollo de su vida universitaria, asesorarlo de manera eficiente y tomando en cuenta la rutina que mantiene a diario.

Propuesta para Medidas de mejora a la Gestión de Apoyo académico en tutorías para el deportista calificado.

- Preferencia en la elección de turno, horarios, grupos, tutorías, para lograr la sincronización de los estudios con los horarios de entrenamientos, la participación en competiciones oficiales y los viajes tanto a nivel nacional como internacional.

- Apoyo en la justificación de faltas en asistencia hasta un límite del 30\% de las sesiones lectivas de carácter obligatorio por participación en competiciones oficiales justificadas mediante certificación de la federación deportiva correspondiente o permiso que se le expida desde la Oficina de Deportes de la misma UNMSM, según sea el caso.

- Implementar facilidades para la flexibilidad del rol de exámenes y de prácticas obligatorias cuando estos ocurran durante las competiciones oficiales justificadas mediante certificación de la federación deportiva a la que pertenece o con constancia de la Oficina de Deportes y avalada por

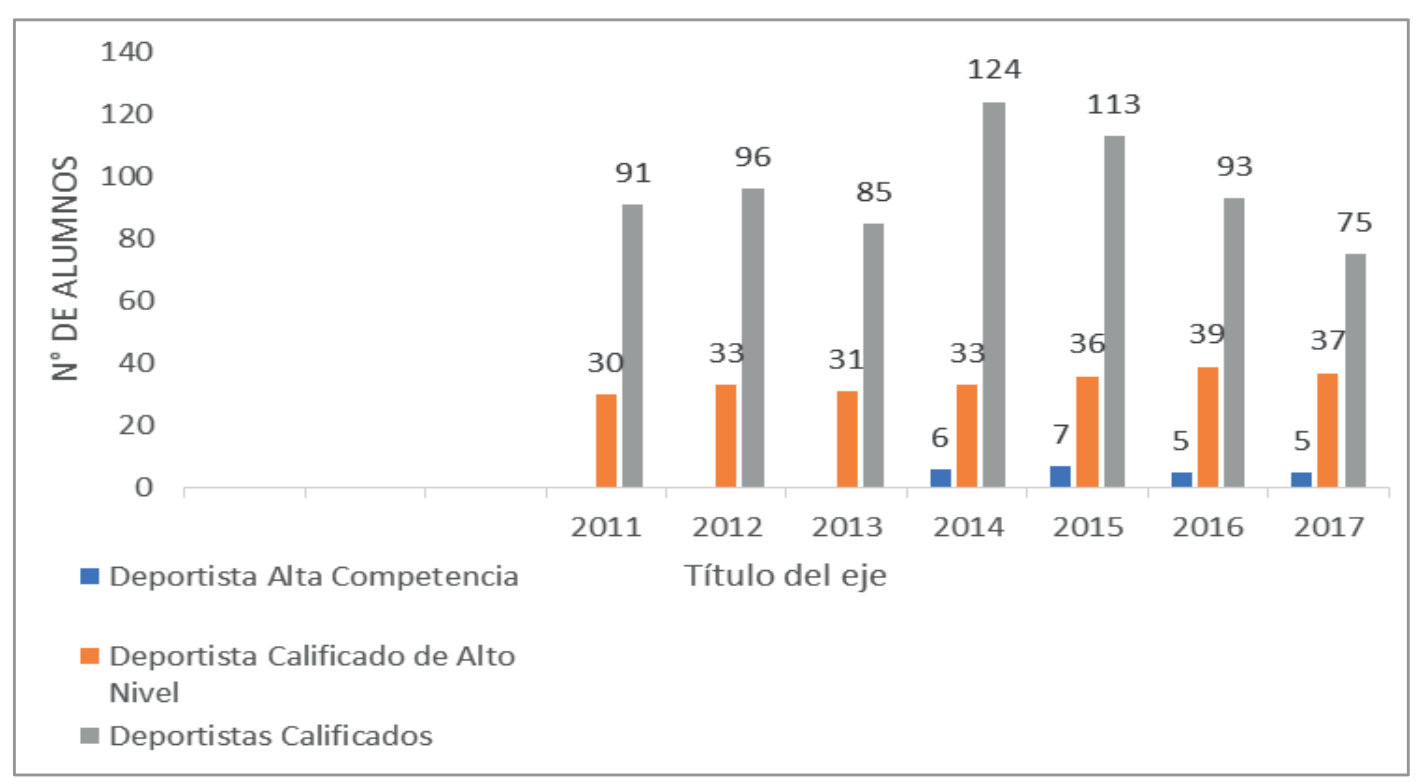

Figura 2. Deportistas Calificados ingresantes 2011 - 2017. Obtenido de la Unidad de Planificación de la UNMSM. 
la Oficina General de Bienestar Universitario (OGBU).

- Entregar una bonificación de 3 créditos académicos por participación en actividades deportivas oficiales del calendario deportivo universitario tal como son los juegos deportivos nacionales universitarios que se realizan cada dos años, designación de un docente tutor(a), quien dará el respectivo apoyo académico y además será el nexo entre el alumno deportista en el programa de tutoría y las diferentes autoridades universitarias (Ver figura 3).

\section{DISCUSIÓN}

En relación a los resultados con el trabajo de investigación (Lagos, 2014), en donde el objetivo se centró en encontrar las causas de deserción en los estudios de los deportistas universitarios en las principales sedes universitarias en Colombia. Donde existen programas de desarrollo deportivo universitario, en los cuales al igual que en el presente trabajo la variable recurrente encontrada en la problemática fue la falta de tiempo del deportista para llevar adecuadamente las clases, la falta de apoyo de las autoridades que dirigen el deporte en las

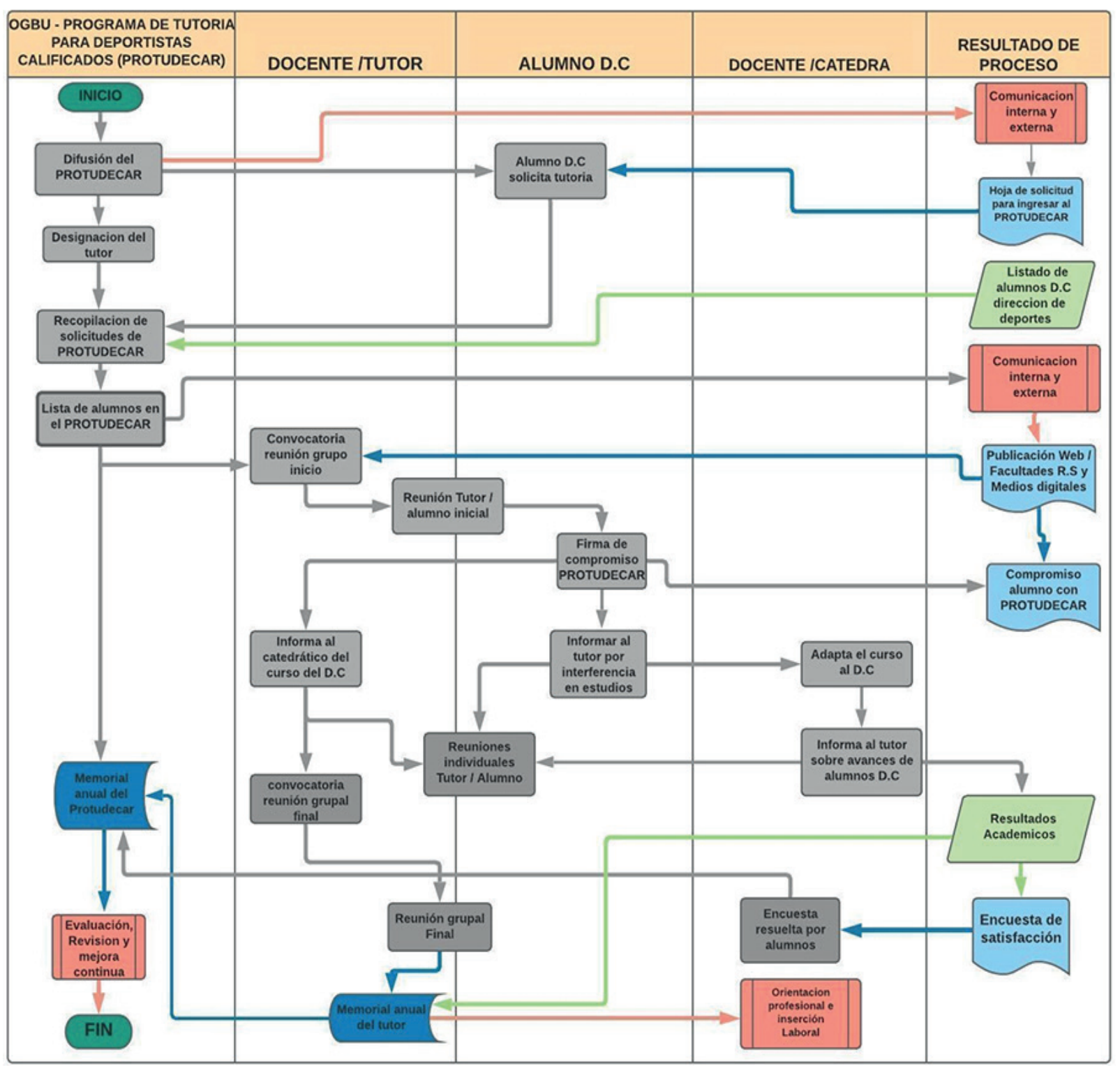

Figura 3. Diagrama de procesos con la solución propuesta para programa de Tutorías académicas a deportistas calificados de la UNMSM.

Elaboración propia. 
universidades, la falta de tolerancia por el trabajo que realizan los deportistas, escasa motivación para armonizar la vida académica con el desarrollo deportivo en los estudiantes. Así mismo contempla una serie de estrategias que pudo extraer entre las respuestas de los entrevistados que le sirvió luego para establecer las conclusiones finales con posibles soluciones. En ambos casos de investigación, se puede concluir que el Estado representado por las universidades nacionales tiene una oportunidad de reiniciar, en base a la calidad, un departamento álgido en un área modelo al implementar la gestión de la calidad en este caso utilizando los preceptos del modelo de Malcolm Baldrige. El deporte hoy en día administrado adecuadamente en manos de personal profesional calificado que entiendan el verdadero valor del deporte y determinado a la sociedad, permite crear una cultura de bienestar, salud y beneficio en todo sentido para quienes lo practican sin distingo del nivel alcanzado, se vislumbra como una herramienta sostenible de desarrollo perdurable en el tiempo para la sociedad.

\section{BIBLIOGRAFÍA}

Acosta, R. (2005). Gestión y administración de organizaciones deportivas. Badalona: Paidotribo.

Álvarez, C. (2011). Metodología de la investigación cuantitativa y cualitativa. Universidad Surcolombiana, Neiva: Facultad de Ciencias Sociales y Humanas de la Universidad Surcolombiana.

Álvarez, P. (2012). Armonización entre el proceso de aprendizaje y práctica deportiva en universitarios deportistas de alto nivel. México: Cultura, Ciencia y Deporte.

Cevidanes, M. (2009). La organización y gestión de las actividades físico deportivas en la universidad: Un caso práctico. Málaga - España: Universidad de Málaga.

Cruz, J. (2012). Modelo de gestión para el desarrollo deportivo en la Universidad Nacional de Colombia sede Bogotá. Bogotá: Universidad Nacional de Colombia.

Dorado, A. (2008). "La gestión del deporte a través de la Calidad". VI Congreso de Agesport Andalucia (Págs. 26 - 29). Andalucía - España: Agesport.
Egaña, Y. (2014). La Gestión de la calidad en el deporte. País Vasco - España: Universidad del País Vasco.

Instituto para la Democracia y la Asistencia Electoral - IDEA Internacional (2008). Gestión pública. Obtenido de http://www2.congreso.gob. pe/sicr/cendocbib/con4_uibd.nsf/8453BD9D9F57489405257C0C0014A7FC/\$FILE/Gesti\%C3\%B3n_P\%C3\%BAblica.pdf

Instituto Peruano del Deporte (2016). Proyecto de política nacional del deporte. Lima: IPD.

Lagos, D. (2014). Estrategias de las universidades colombianas para favorecer el acceso, permanencia y finalización de estudios en deportistas de alto nivel. Santander - Colombia.

Meynaud, J. (1972). El deporte y la política. . Barcelona - España: Hispano Europea.

Miller, R. (2012). El fútbol como negocio. Lima: Pacífico.

Pérez, J. (2014). Metodología y diseño de la investigación científica. Lima: Universidad

Pontificia Universidad Católica del Perú. (2014). Congreso grandes retos de la gestión deportiva en el Perú. Lima: PUCP.

Redondo, S. y Contreras, J. (2015). Necesidades académicas de los deportistas de alto nivel en el instituto distrital para la recreación y deporte. Bogotá, Colombia: Universidad Santo Tomás, Facultad de Cultura Física, Deporte y Recreación.

Tamayo, T. (2010). El proceso de la investigación Científica. México: Limusa.

Torregrosa, M., Sánchez, X. y Cruz, J. (2004, octubre). El papel del psicólogo del deporte en el asesoramiento académico - vocacional del deportista de Élite. Revista de psicología del deporte. Mensual. Recuperado de https://ddd.uab.cat/pub/ revpsidep. pp. 215-228

Universidad Nacional Mayor de San Marcos. (2013). http://www.unmsm.edu.pe. Obtenido de http:// www.unmsm.edu.pe/archivos/md_unmsm_ polideportivo.pdf 
